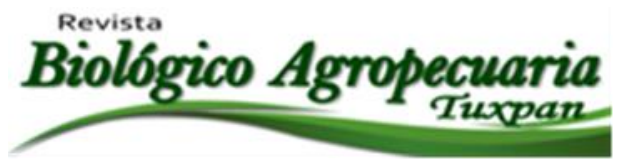

\title{
Evaluación económica y clínica de la técnica quirúrgica modificada de traslape en hernia umbilical bovina
}

Economic and clinical evaluation of the surgical technique of overlapping in bovine umbilical hernia.

Arieta-Román Ronnie de Jesús ${ }^{1 凶}{ }^{凶}$, Rodríguez-Orozco Nereida ${ }^{2}$, Graillet-Juárez Eduardo ${ }^{1}$ Retureta Aponte Alejando ${ }^{1}$ Fernández Figueroa José, Antonio ${ }^{1}$ Martínez Martínez Marina ${ }^{1}$

${ }^{1}$ Facultad de Ingeniería en Sistemas de Producción Agropecuaria. Universidad Veracruzana Carretera Costera del Golfo km 220, Col. Agrícola Michapan. Acayucan, Veracruz. C.P. 96100. ${ }^{2}$ Facultad de Ciencias Agrícolas. Universidad Veracruzana. Lomas del Estadio s/n Xalapa, Veracruz, México C.P. 91000.

${ }^{\circledR}$ Autor para correspondencia: roarieta@uv.mx

Recibido: $14 / 07 / 2020$

Aceptado: 19/09/2020

\section{RESUMEN}

El objetivo fue evaluar clínica y económicamente la técnica quirúrgica modificada de traslape para corrección de hernia umbilical como estrategia productiva en empresas de producción bovina. La investigación fue de tipo experimental descriptivo. Se utilizaron 15 bovinos (2 machos y 13 hembras), peso promedio $73 \mathrm{~kg}$, anillo herniario con diámetro $\leq 10 \mathrm{y} \geq 6$ centímetros. Se realizó incisión elíptica con extensión de 1-2 cm en craneal y caudal respecto al anillo herniario; se continuó por las capas del tejido conectivo areolar hasta el saco herniario, se diseccionó y dejó libre hasta el margen del anillo. Posteriormente se reavivaron los bordes del anillo herniario con corte extendido de 1-1.5 cm alrededor de éste. El cierre de músculos abdominales fue mediante suturas Mayo modificadas de superposición (traslape). Se elaboraron puntos de sutura suficientes hasta la reducción total del anillo herniario. La piel se cerró con sutura interrumpida con nylon. Los indicadores demuestran que la técnica quirúrgica modificada de traslape fue eficiente al obtener una alta Relación Costo Beneficio de 4.6, y una mejoría de los becerros a los 24 días para su función zootécnica.

Palabras clave: Traslape, costo-beneficio, hernioplastia umbilical, anillo herniario.

\begin{abstract}
The objective was to evaluate clinically and economically the surgical technique of overlapping to correct umbilical hernia as a productive strategy in bovine production companies. The investigation was of a descriptive experimental type. 15 bovines ( 2 males and 13 females), average weight $73 \mathrm{~kg}$, hernial ring with diameter $\leq 10$ and $\geq 6$ centimeters were used. An elliptical incision with a cranial and caudal extension of 1-2 cm was made with respect to the hernial ring; it was continued through the layers of the areolar connective tissue to the hernial sac, dissected and left free until the margin of the ring.
\end{abstract}


Subsequently, the edges of the hernial ring were revived with an extended cut of $1-1.5 \mathrm{~cm}$ around it. The abdominal muscles were closed using modified Mayo overlapping sutures. Sufficient stitches were made until total reduction of the hernial ring. The skin was closed with suture interrupted with nylon. The indicators show that the overlapping surgical technique was efficient, obtaining a high Cost Benefit Ratio of 4.6, and an improvement in the calves at 24 days for their zootechnical function.

Keywords: Overlap, cost-benefit, umbilical hernioplasty, hernial ring.

\section{INTRODUCCIÓN}

La hernia umbilical es la salida de un asa intestinal por un punto débil del anillo umbilical debido a un defecto en el cierre de la pared abdominal. Esta situación generalmente se diagnostica en becerros recién nacidos, con un índice de heredabilidad del 1 al 3\%. En los centros de producción intensiva la incidencia puede alcanzar hasta un 5 u 8\%. (Bailón et al., 2013)

Las hernias umbilicales "congénitas", es decir presentes al nacimiento, se manifiestan en las primeras semanas de vida. Su incidencia se incrementa por la consanguinidad y la predisposición genética, probablemente poligénica, con la participación de influencias ambientales modulantes (Bailón et al., 2018). En la práctica de cirugía profesional en grandes animales es común el hallazgo de las hernias. El éxito del tratamiento quirúrgico depende del diámetro del anillo, de la cantidad de contenido, de la resistencia de los tejidos localizados en los bordes del anillo, inflamación local, edad, peso del animal, manejo alimenticio y cuidados postoperatorios (Nguhiu et al., 1991).

La hernia umbilical es una patología quirúrgica con alta recidiva, debido al mal posoperatorio (infecciones, dieta, reposo, etc.) y a la falta de opciones en aquellos animales de anillos herniarios de gran tamaño que son intervenidos con la técnica tradicional. Para estos casos de hernias grandes y complicadas, la variante está en el tipo de punto utilizado para cerrar el anillo pos reintroducción del contenido, por lo que pueden emplearse diferentes técnicas quirúrgicas de corrección para esta patología (Rings, 1995).

En este contexto una investigación realizada por Arieta, et al. (2017) muestra la eficacia en la utilización de una malla de propileno para la corrección de hernias umbilicales en animales grandes dentro de la empresa bovina, con una relación beneficio costo para el empresario ganadero que opta por la cirugía de 3.8, siendo evidente la recuperación del costo de la inversión y el beneficio adicional de 2.8 .

Sin embargo, esta técnica es eficiente para animales que presentan anillos herniales de gran tamaño, por lo que es indispensable contar con una alternativa quirúrgica que sirva tanto para tratar este tipo de hernias complicadas, como para corregir hernias umbilicales recidivantes intervenidas por el método tradicional. Bajo este esquema, el objetivo del presente estudio fue el evaluar clínica y económicamente la técnica quirúrgica modificada de traslape para corrección de hernia umbilical como estrategia productiva en empresas de producción bovina.

\section{MATERIALES Y MÉTODOS}

La investigación es de tipo experimental descriptivo. Se realizó en unidades de producción bovina ubicados en la zona norte y sur del estado de Veracruz., en el período comprendido entre marzo 2019 a marzo 2020. 
Se utilizaron 15 bovinos (2 machos y 13 hembras), razas (11 Brahman, 3 Beefmaster y 1 Suizo Americano) con edades entre los 2 y 6 meses, peso promedio de $73 \mathrm{~kg}$ y anillo herniario con diámetro $\leq 10 \mathrm{y} \geq 6$ centímetros.

Hallazgos al examen clínico. Los animales seleccionados para la intervención quirúrgica presentaron en la inspección general buena condición corporal $(3.0 \pm 0.5$, en una escala de
1-5), temperamento dócil y a nivel umbilical una neoformación sin evidencia de inflamación activa, reducible y blanda a la palpación, con la que se determinó también la presencia de anillo herniario. A la auscultación se diagnosticó ruidos intestinales en la saculación umbilical de todos los animales, lo que permitió la confirmación diagnóstica de hernia umbilical. (Figuras 1, 2 y 3 ).

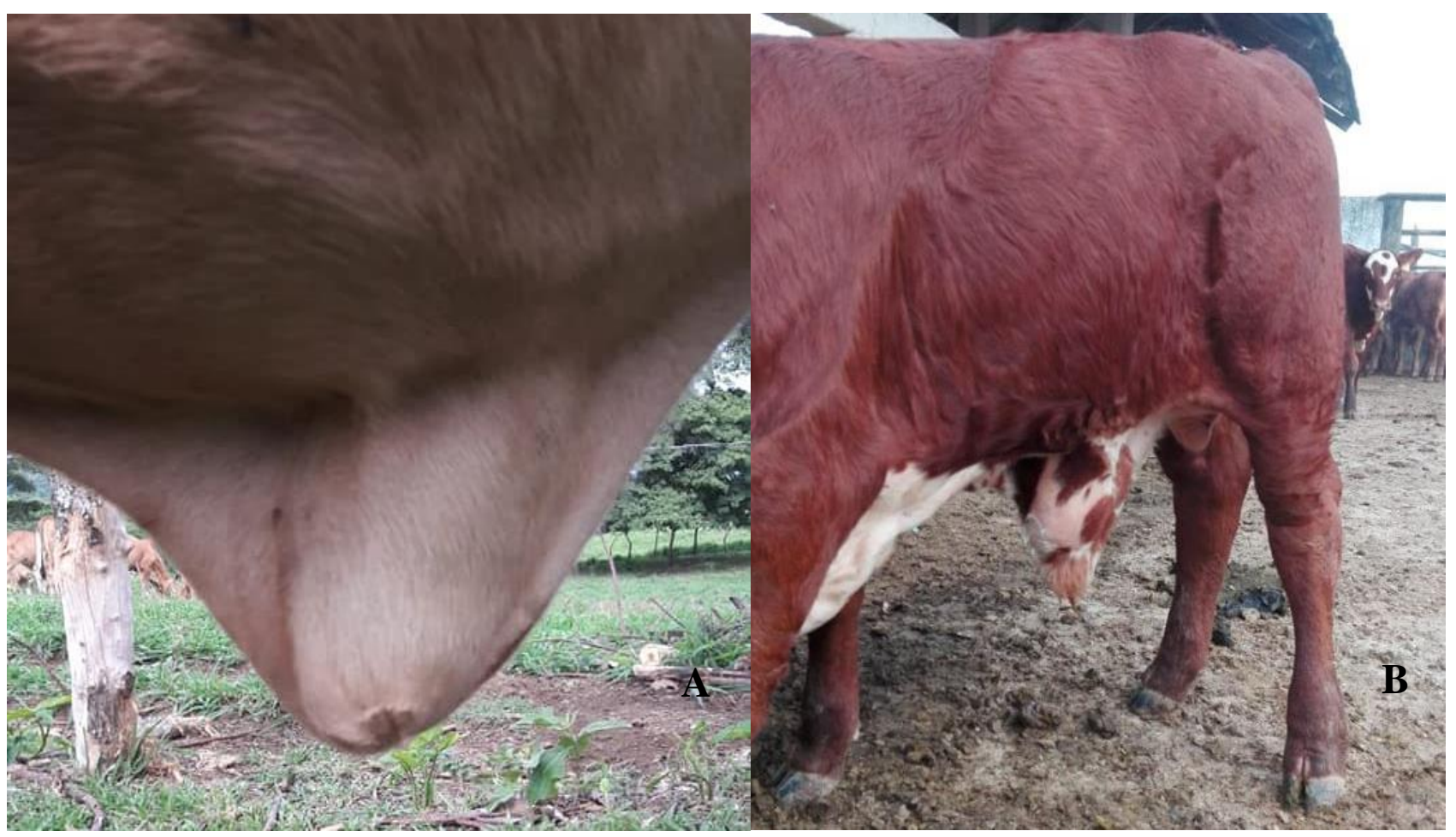

Figura 1. Hernia en hembra bovina. (A). Hernia en macho bovino (B). 


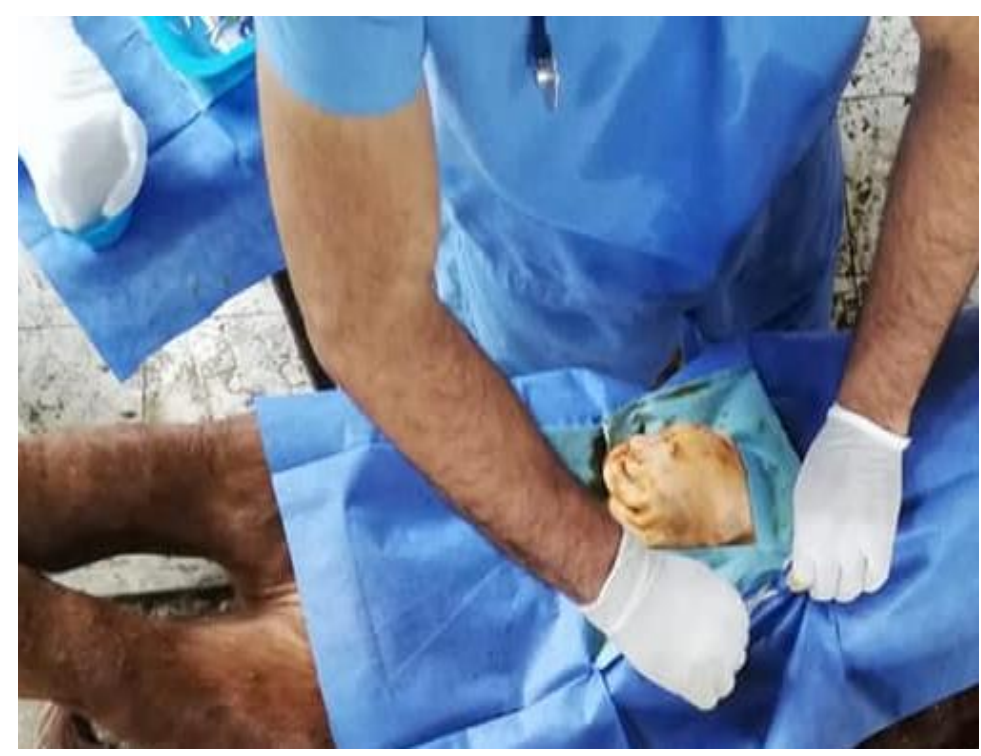

Figura 2. Palpación de anillo herniario.

Procedimiento preoperatorio. Se consideró un ayuno de 24 horas de alimento y 8 horas de agua, se procedió a tranquilizar al animal con clorhidrato de xilacina en dosis de 0.1 a $0.2 \mathrm{mg}$ por $\mathrm{kg}$ de peso vivo. Antisepsia del saco herniario y región umbilical. La piel del área operatoria se lavó con jabón quirúrgico, depiló y embrocó dos veces con gasas saturadas de yodo, frotándose vigorosamente. Se realizó un bloqueo de campo sin infiltrar la zona de incisión de manera circular siguiendo el patrón del saco herniario a unos $5 \mathrm{~cm}$ de la línea de incisión en dirección proximal. Para este bloqueo se empleó clorhidrato de lidocaína al $2 \%$ a dosis de $2 \mathrm{ml}$ en cada sitio de infiltración. En el presente estudio se utilizaron 6 puntos de infiltración, 3 de cada lado (Arieta et al., 2017).

Procedimiento quirúrgico. Siguiendo la técnica quirúrgica de traslape descrita por Bailón et al. (2018). se realizó una incisión elíptica con una extensión de 1-2 cm en craneal y caudal con respecto al anillo herniario. La incisión se continuó a través de las numerosas capas del tejido conectivo areolar hasta el saco herniario, el cual se diseccionó y se dejó libre hasta el margen del anillo. Posteriormente se reavivaron los bordes del anillo herniario haciendo un corte, el cual se extendió de 1-1.5 cm alrededor de éste. Durante la intervención se aplicó frecuentemente una solución de suero salino fisiológico con oxitetraciclina, con el fin de lavar y mantener hidratados los tejidos de la región.

El cierre de los músculos abdominales se llevó a cabo mediante una serie de suturas Mayo modificadas de superposición (traslape) (Figuras 4 y 5). Para este propósito se utilizó material de sutura no absorbible, liso, como nylon No. 6 multifilamento recubierto (Supramid, Braun Melsingen AG) o nylon monofilamento. Se elaboraron los puntos de sutura suficientes hasta la reducción total del anillo herniario. Para evitar la punción accidental de una víscera, se realizaron las suturas sin que hubiera riesgo de puncionar alguna de éstas, colocando un dedo en el margen del anillo herniario y comprimiendo el saco contra la pared. La tracción simultánea de la sutura superpuso los bordes reavivados del anillo y se mantuvo mientras cada punto de sutura se anudó en forma individual. Seguido de ellos se colocaron puntos en $\mathrm{U}$ de refuerzo. El tejido conectivo subcutáneo se cerró con una sutura continua simple usando poliglactina 910 (Vicryl, Ethicon) y la piel se cerró con una sutura interrumpida con nylon (Figura 6). 


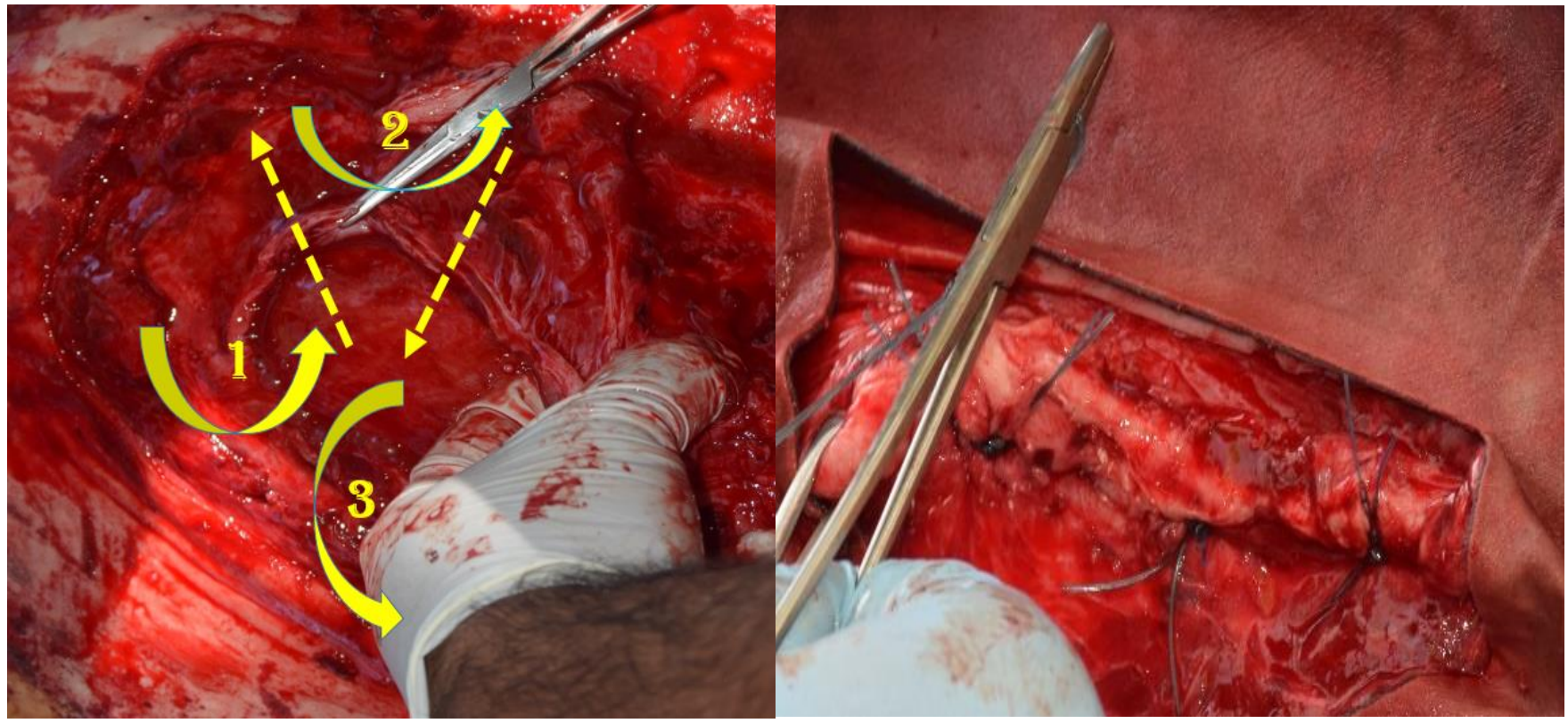

Figura 3. Trayecto de la sutura de traslape (A). Sutura finalizada de traslape (B)

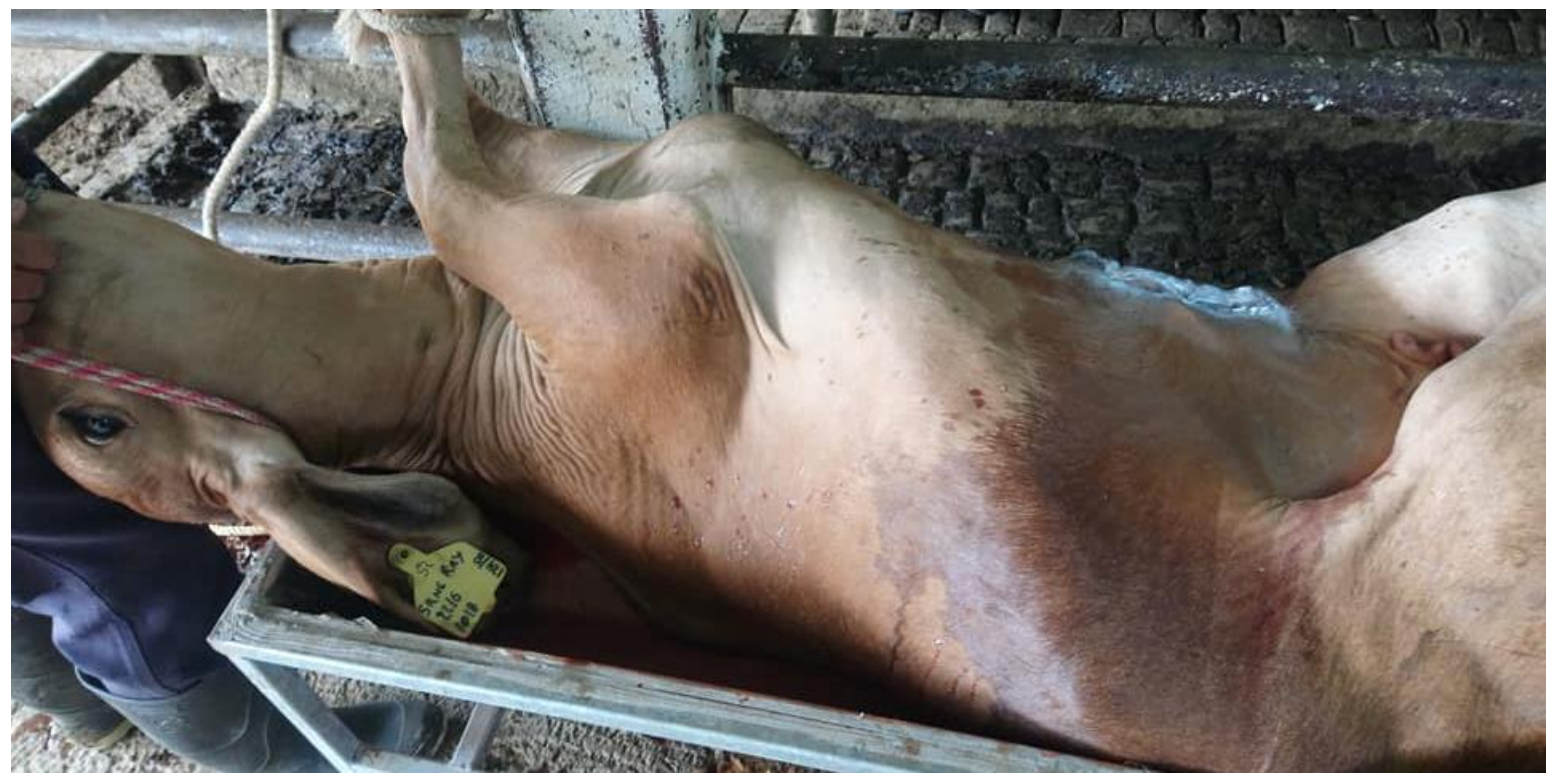

Figura 4. Sutura de piel.

\section{Posoperatorio.}

La recomendación postoperatoria, incluyó penicilina G procaína a dosis de 20000 UI / kg cada $12 \mathrm{~h}$ por 5 días, vía intramuscular profunda, además de curaciones locales, durante 7 días, por último, se retiraron los puntos el día 12 posoperatorio.

\section{Evaluación clínica:}

Tiempo de la operación: Se estimó desde el inicio del acto quirúrgico hasta el final del mismo.

Traumatismo operatorio: Se valoró midiendo las líneas de incisión para realizar el procedimiento quirúrgico. 
Recuperación posoperatoria: Se evaluó desde el término de la cirugía hasta la recuperación total del paciente.

Procesos infecciosos: Se practicaron diariamente exámenes físicos y generales a cada paciente para detectar algún proceso infeccioso posoperatorio.

Relación Beneficio Costo (RBC): Se calculó dividiendo el valor actualizado de los beneficios (ingresos) entre el valor actualizado de los costos (egresos). Se consideraron los siguientes referentes para el cálculo ${ }^{1}$ :

Precio por Kg de becerro en pie: 1,94 USD

Precio por $\mathrm{Kg}$ de becerro en pie con hernia umbilical: 0.97 USD

Peso promedio del becerro: $250 \mathrm{Kg}$

Costo de la cirugía: 105,51 USD

Con base en los datos anteriores se estimó un ingreso por la venta de un bovino sano en pie de 485,33 USD obteniendo una RBC de 4.6, mientras que el ingreso estimado por la venta de un bovino con hernia umbilical es de 242,67 USD ya que el mercado reduce el precio en un $50 \%$ por esta patología, el cual es menor aun considerando el costo de la cirugía.

\section{RESULTADOS Y DISCUSIÓN}

En los resultados del presente estudio se obtuvo un tiempo promedio por cirugía de 15 minutos, con un traumatismo operatorio de una primera incisión circular a nivel saco herniario en promedio de $12 \mathrm{~cm}$ de diámetro referente a la línea media. La inflamación intensa se presentó en los bovinos intervenidos quirúrgicamente, lo que se atribuye a una reacción normal por el manejo delicado de los tejidos durante la cirugía. En relación con la recuperación posoperatoria promedio fue de 24 días, con monitoreo cada 24 horas, no se presentó proceso infeccioso alguno y a los 15 bovinos intervenidos quirúrgicamente les funciono la técnica quirúrgica de traslape. En un estudio similar en el Departamento de Córdoba, Colombia se empleó fascia abdominal aguda para hernioplastía umbilical reportando inflamación intensa en el $80 \%$ de los animales, que posiblemente se debió a un mayor tiempo de exposición al que fueron sometidos los locales quirúrgicos, lo que implica un mayor tiempo de anestesia, menor irrigación y oxigenación de los tejidos expuestos, pérdida de temperatura local, así como un mayor riesgo de contaminación (Nguhiu et al., 1991). Además, el presente estudio tiene similitud al trabajo mencionado referente a la inflamación, donde se presentó en el $66.6 \%$ de los animales operados.

En lo que respecta a la Relación Beneficio/Costo con la utilización de la técnica quirúrgica se obtuvo un indicador de 4.6 lo que indica la recuperación del costo invertido y un beneficio económico de 3.6 adicional. Esto es evidente ya que con la intervención quirúrgica el precio del mercado se mantiene y permite recuperar la inversión realizada, lo cual es superior al ingreso obtenido por la venta de un animal con la patología.

\section{CONCLUSIÓN}

Los indicadores muestran que la técnica quirúrgica modificada de traslape fue eficiente económica y técnicamente, al obtener una RBC de 4.6 que muestra la recuperación de la inversión realizada y un beneficio económico adicional, así como una recuperación de los becerros en un lapso de 24 días para su función zootécnica.

\footnotetext{
${ }^{1}$ En México el precio por $\mathrm{Kg}$ de becerro sano en pie es de $\$ 46.00 \mathrm{MXN}$ y de $\$ 23.00 \mathrm{MXN}$ para becerros en pie con hernia umbilical.
} 


\section{LITERATURA CITADA}

Arieta R.R.J., Olivera G.D.A., Rodríguez O.N., Fernández F.J.A. 2017. Evaluación clínica y RBC de la hernioplastía umbilical utilizando malla quirúrgica de propileno en la empresa bovina. REDVET - Revista electrónica de Veterinaria. 18 (5): 1-7. ISSN:

1695-7504.

http://www.veterinaria.org/revistas/redvet /n050517.html

Bailón B.A., Olguín y Bernal A.F., Martínez L.A. 2013. Tratamiento Quirúrgico de Hernias Umbilicales en Bovinos Mediante el Uso de Material Protésico. Engormix. https://www.engormix.com/ganaderialeche/articulos/tratamiento-quirurgicohernias-umbilicales-t30530.htm
Nguhiu MJA, Munyua SJM, Mbithi PFM, Mbiuki SM, Mogoa EGM. 1991. How to improve the prognosis of ventral abdominal hernias in large animals: "modified" overlapping technique. Bull Anim Health Prod Afr; 39: 315-320. http://erepository.uonbi.ac.ke/handle/112 95/11028

Rings M. 1995. Umbilical Hernias, Umbilical Abscesses and Urachal Fistulas: Surgical Considerations. Veterinary Clinics of North America: Food Animal Practice. 11 (1): 137-148.

https://doi.org/10.1016/S07490720(15)30512-0

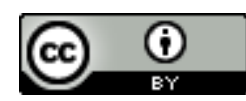

Este texto está protegido por una licencia Creative Commons $\underline{4.0}$.

\footnotetext{
Usted es libre para Compartir — copiar y redistribuir el material en cualquier medio o formato-y Adaptar el documento — remezclar, transformar y crear a partir del material - para cualquier propósito, , incluso para fines comerciales, siempre que cumpla la condición de:

Atribución: Usted debe dar crédito a la obra original de manera adecuada, proporcionar un enlace a la licencia, e indicar si se han realizado cambios. Puede hacerlo en cualquier forma razonable, pero no de forma tal que sugiera que tiene el apoyo del licenciante o lo recibe por el uso que hace de la obra.

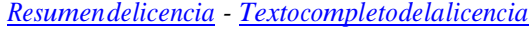

